Nathaniel W. Yang, MD

Department of Otorhinolaryngology College of Medicine - Philippine General Hospital University of the Philippines Manila

Department of Otolaryngology Head and Neck Surgery

FEU-NRMF Institute of Medicine

Department of Otorhinolaryngology Head and Neck Surgery

The Medical City
Correspondence: Dr. Nathaniel W. Yang

Department of Otorhinolaryngology

Ward 10, Philippine General Hospita

University of the Philippines Manila

Taft Avenue, Ermita, Manila 1000

Philippines

Phone: (632) 5264360

Fax: (632) 5255444

Email: nwyang@gmx.net

\section{Spontaneous Middle Fossa Encephalocele}

A 48-year-old man presented with a unilateral right hearing loss of four months' duration. A right middle ear effusion was noted on physical examination. Endoscopic examination of the nasopharynx was unremarkable. Due to the duration of the symptoms, myringotomy with ventilation tube insertion was offered as a treatment option. Upon myringotomy, clear pulsatile liquid flowed out of the incision. More than $5 c c$ of liquid was collected which continued to flow out despite active suctioning. Due to the realization that the liquid most likely represented cerebrospinal fluid, insertion of a ventilation tube was not performed. The ear canal was packed with sterile cotton, and the patient was given a short course of acetazolamide to decrease CSF production. Upon further questioning, the patient did not have any prior head trauma. The patient then underwent both computerized tomographic (CT) imaging and magnetic resonance imaging (MRI) of the temporal bone to look specifically for evidence of a dehiscence in the middle fossa plate (tegmen) or posterior fossa plate, as well as the presence of a meningoencephalocele.

Computerized tomographic imaging of the temporal bone in the axial plane showed a soft tissue density completely occupying the air-containing spaces of the middle ear, epitypanum and mastoid air cells without any evidence of bony erosion of the scutum, the ossicles, or the bony septations of the mastoid air cells. T2-weighted magnetic resonance imaging in the axial plane showed that the soft tissue densities in the middle ear, the epitympanum and mastoid air cells had a naturally high signal intensity characteristic of fluid. (Figure 1)

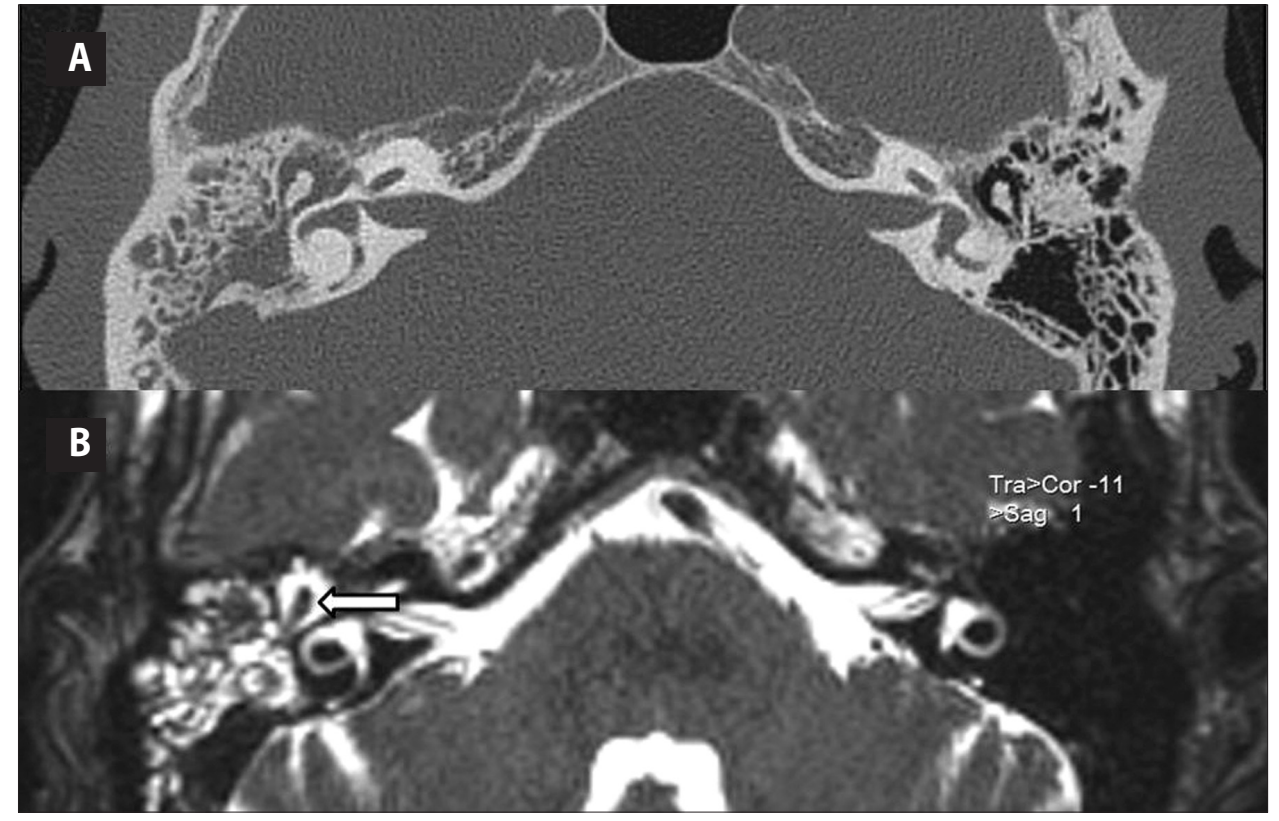

Figure 1. CT imaging in the axial plane. A. At the level of the head of the malleus, incus body and lateral semicircular canal shows homogenous soft tissue density in the right epitympanum and mastoid air cell system. Note that the bony septations are intact and are similar to that on the uninvolved left side. T2-weighted MR imaging at the same level in the axial plane. B. The high signal intensity of the soft tissue densities similar to the CSF in the cerebellopontine angle cisterns. The ossicles (white arrow with black outline) can be clearly seen within the fluid-filled epitympanum. 
On coronal CT imaging, a dehiscence of the middle fossa plate (tegmen) was noted lateral to the superior semicircular canal. Magnetic resonance imaging in the same plane revealed a soft tissue density in the region of the dehiscence that was contiguous with, and isointense with the temporal lobe. This soft tissue density appeared to originate from the temporal lobe, and extended downwards into the upper portion of the mastoid antrum. No enhancement was noted on gadolinium-enhanced T1-weighted imaging. (Figure 2) With these imaging findings, a middle fossa encephalocele was considered. Exploratory mastoidectomy confirmed the diagnosis, and the patient subsequently underwent a transmastoid repair of the tegmen and dural dehiscence using both temporalis fascia and mastoid cortical bone after the herniated brain tissue was amputated.
A middle fossa encephalocele is a condition of the temporal bone that may arise as a complication of chronic otitis media, temporal bone fractures, or after surgery involving the temporal bone. Although rare, spontaneous middle fossa encephaloceles may also occur. ${ }^{1,2}$ One must maintain a high degree of clinical suspicion for this condition in an adult patient presenting with a unilateral middle ear effusion or watery otorrhea in the absence of an identifiable cause of otologic disease ${ }^{2}$ or nasopharyngeal pathology. It should definitely be highly considered if profuse, persistent clear otorrhea is encountered during a myringotomy for what may initially appear to be a chronic middle ear effusion. Surgical treatment of the encephalocele and repair of the skull base defect is generally recommended, as life threatening complications such as meningitis, brain abscess and temporal lobe seizures have been known to occur. ${ }^{2}$

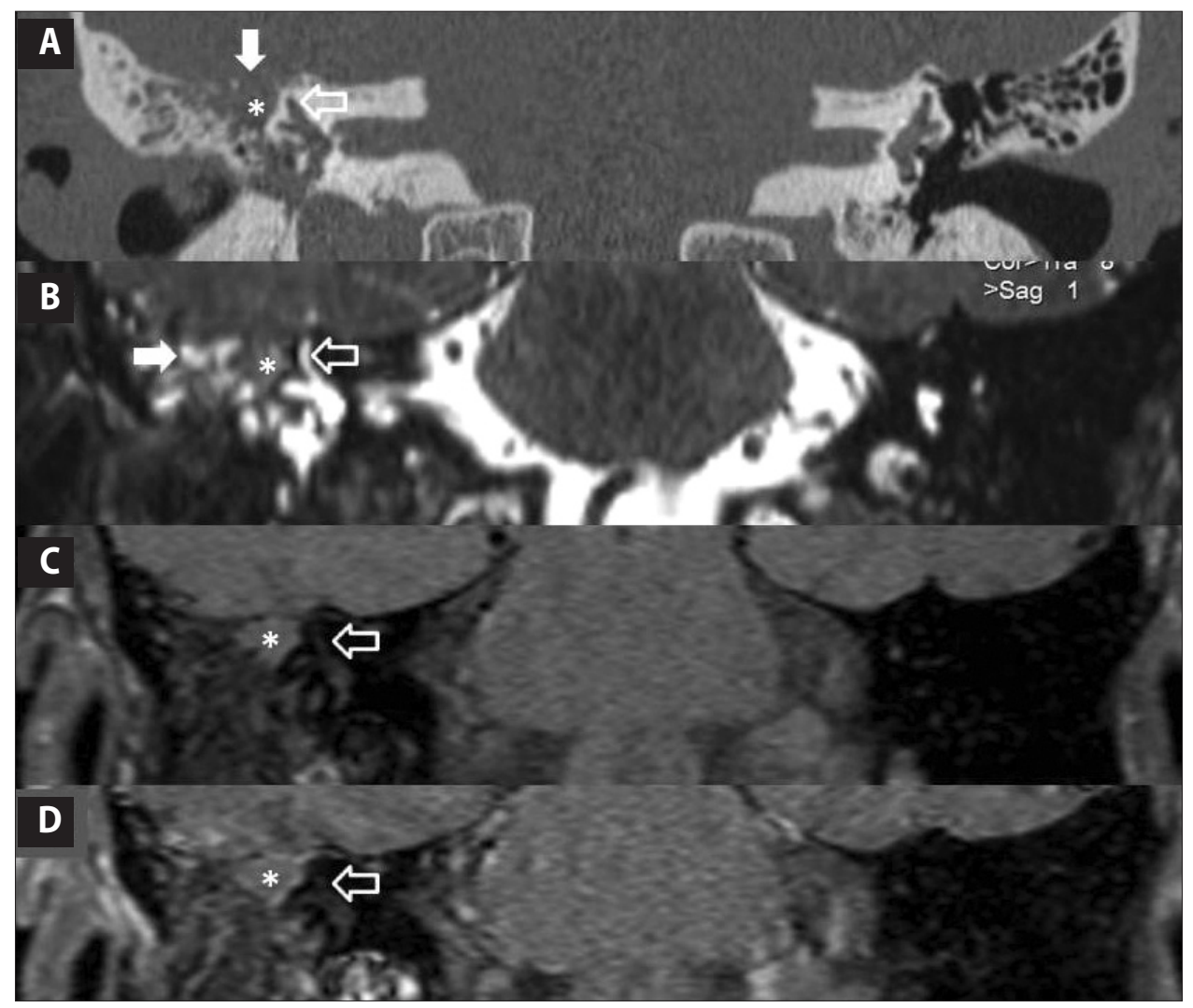

Figure 2. CT and MR imaging in the coronal plane at the level of the superior semicircular canal (white outlined arrow, all images). CT imaging. A. A dehiscence in the tegmen mastoideum (white solid arrow). T2-weighted MR imaging. B. The high signal intensity of the fluid in the mastoid air cells (white solid arrow). The soft tissue density seen directly under the tegmen dehiscence in all of the images (white asterisk) has the same signal intensity as the overlying brain in all of the MR sequences. This soft tissue density is directly connected to the overlying temporal lobe in both the T2-weighted image. B. and the non-contrast enhanced T1-weighted image. C. No enhancement is seen in the Gd-enhanced T1-weighted image $\mathbf{D}$.

\section{REFERENCES}

1. Rao AK, Merenda DM, Wetmore SJ. Diagnosis and management of spontaneous cerebrospinal fluid otorrhea. Otol Neurotol. 2005 Nov; 26(6): 1171-1175. PMID: 16272936.

2. Gubbels SP, Selden NR, Delashaw JB Jr, McMenomey SO. Spontaneous middle fossa encephalocele and cerebrospinal fluid leakage: diagnosis and management. Otol Neurotol. 2007 Dec; 28(8): 1131-1139. DOI: 10.1097/MAO.0b013e318157f7b6 PMID: 17921911. 